\title{
Spine Surgical Procedures during Coronavirus Disease 2019 Pandemic: Is It Still Possible to Take Care of Patients? Results of an Observational Study in the First Month of Confinement
}

\author{
Mikael Meyer, Solène Prost, Kaissar Farah, Jean-Baptiste Denis, \\ Henry Dufour, Benjamin Blondel, Stéphane Fuentes \\ Unité de Chirurgie Rachidienne, Aix-Marseille Université, APHM, CNRS, ISM, CHU Timone, Marseille, France
}

\begin{abstract}
Study Design: Observational study.
Purpose: The actual sanitary crisis led to a massive mobilization of the sanitary system toward intensive care units and management of coronavirus disease 2019 (COVID-19) patients. However, some patients still require spinal interventions. The present study aimed to assess the impact of the COVID-19 pandemic on spine surgical in a moderate COVID-19 cluster region.

Overview of Literature: Previous studies have reported screening and management of patients with spinal conditions during the COVID-19 pandemic. However, to date, knowledge, no observational study on spine surgeries during the pandemic has not been reported.

Methods: Between March 17, 2020 and April 17, 2020, information on spine surgical activity was prospectively collected at our institution. This surgical activity related to the first month of confinement in France was compared to the activity during the same period in 2019 to evaluate the impact of the COVID-19 pandemic on surgical activities.

Results: In order to reduce the contamination rate of patients and medical staff during hospitalization, the spine department was completely reorganized. Non-urgent elective spine surgeries were cancelled. When considering the global amount of surgeries procedures during the first month of confinement, a decrease of almost $50 \%$ was observed in the number of surgical procedures. During the study period, 62 patients were eligible for spine surgery. The numbers of patients managed for tumor and infectious cases were stable, while a considerable reduction was observed in the number of trauma and degenerative cases. During the follow-up period, two patients were tested as COVID+ during the postoperative course, and no cases of medical or paramedical staff contamination were reported using polymerase chain reaction-testing.

Conclusions: During the COVID-19 pandemic, it is possible to maintain spine surgical activity. Each surgical procedure must be discussed and organized with all the caregivers involved. Indications for surgery must be in line with the scientific guidelines and adapted to each healthcare facility.
\end{abstract}

Keywords: Spine; COVID-19; Organizations; Surgery

Received Apr 28, 2020; Accepted Apr 28, 2020

Corresponding author: Stéphane Fuentes

Unité de Chirurgie Rachidienne, CHU Timone, 264 rue Saint Pierre, 13005 Marseille, France

Tel: +33-4-9138-8692, E-mail: sfuentes@ap-hm.fr 


\section{Introduction}

Since the beginning of the coronavirus disease 2019 (COVID-19) pandemic in December in China, the first positive patient in France was reported on January 24, 2020. With a rapidly evolving situation, a declaration of a pandemic situation was made by the World Health Organization on March 11, 2020 [1]. A national emergency plan was adopted in France [2] and finally national confinement was ordered on March 17, 2020.

According to the guidelines from the health authorities [3], elective surgeries were cancelled, and only urgent surgeries were performed. In order to help spine physicians, the French Spine Society (SFCR) dictated guidelines using a three-level scale (urgent, semi-urgent, and non-urgent procedures) for establishing surgical priorities [4].

The present study was designed to assess the impact of the COVID-19 pandemic on spine surgical activities in our department and its associated organization in a moderate COVID-19 cluster region.

\section{Materials and Methods}

In the duration from March 17, 2020 to April 17, 2020, information on spine surgical activity was prospectively collected in our institution (neurosurgery department and spine unit). For each patient, the preoperative COVID status was tested using polymerase chain reaction (PCR)testing, and the eventual change in the status was noted during hospitalization. For patients with symptoms, such as fever, cough, and dyspnea, and those who were suspected of being infected, a chest computed tomography (CT) scan was obtained.

With regard to the spinal procedure, the following parameters were noted: etiology of the spinal condition, surgical strategy (conventional or minimal-invasive), and surgical team (junior or senior surgeon). During the follow-up period, potential contaminations in patients as well as medical and paramedical staff were investigated.

Finally, this surgical activity related to the first month of confinement in France was compared to the activity during the same period in 2019 to perform a comparison to evaluate the impact of COVID-19 pandemic on the surgical activities.

\section{Results}

\section{Perioperative organization}

In order to reduce the contamination rate in patients and medical staff during hospitalization, complete reorganization of the spine department was performed on March 16, 2020.

All hospital rooms were changed from double to single occupancy rooms, leading to a decrease in resources from 61 to 30 beds. The operation theater was separated into two different zones with a variable number of operatives, as per the evolution of the pandemic. During the first week of confinement, one surgical ward for COVID+ patients and two for COVID- patients with separate pathways were available, and according to the pandemic evolution, one COVID- room was closed during the subsequent 3 weeks.

Each patient wore a surgical facemask before and after the operation. In order to preserve the surgical resources, the surgical team was split into two and assured rotations every half-week.

With regard to intensive care unit (ICU) beds, our institution markedly increased the number of beds from 62 before the pandemic to 126 after the pandemic. Among these 126 beds, 78 were dedicated to COVID+ patients, while 48 were dedicated to COVID- patients in order to maintain urgent cases.

\section{Surgical data}

First, on March 16, 2020, 93 non-urgent elective spine surgeries that were scheduled for April 2020 were cancelled. All the patients who were admitted from this date onward underwent PCR-test for COVID-19 and if symptomatic or suspected, a low-dose COVID protocol chest-CT scan was obtained.

There was a reduction of almost $50 \%$ in the number of surgeries performed worldwide during the first month of confinement (Table 1) with a stable numbers of brain procedures but an important decrease of spine surgeries (56\%).

During the study period, 62 patients were eligible for spine surgery with indications as per those described in the SFCR guidelines. Globally, 1/3rd of the patients were recruited via the emergency department, $1 / 3$ rd from the outpatient clinics, and 1/3rd were referred from others 
Table 1. Global amount and etiologies of surgical procedures during the same period of time (March 17 to April 17) in 2019 and 2020

\begin{tabular}{lrr} 
& \multicolumn{2}{c}{ Year } \\
Surgical procedures & 2019 & 2020 \\
\cline { 2 - 3 } & 149 & 66 \\
Spine & 66 & 57 \\
Brain & 14 & 3 \\
Hypophysis & 229 & 126 \\
\hline Total & & \\
\hline
\end{tabular}

Table 2. Number of patients managed surgically and related etiologies during the same period of time (March 17 to April 17) in 2019 and 2020

\begin{tabular}{lrr} 
& \multicolumn{2}{c}{ Year } \\
\cline { 2 - 3 } Etiology & 2019 & 2020 \\
Tumor & 15 & 15 \\
Trauma & 36 & 5 \\
\hline Degenerative & 80 & 36 \\
Infection & 8 & 6 \\
\hline Total & 149 & 62 \\
\hline
\end{tabular}

hospitals.

The numbers of patients managed for tumor and infectious cases were stable, while that for patients with trauma and degenerative cases decreased considerably. The etiology of spine conditions and comparison with the situation during the same month in 2019 are summarized in Table 2.

With regard to spine surgeries, the following three subgroups of procedures were established: simple procedures, intermediate procedures, and complex procedures (Tables $3-5)$.

Among simple procedures, the number of cases managed for lumbar disk herniation remained stable, while an important decrease was noted in the cases of cervical disk herniation and lumbar canal narrowing. Among intermediate procedures, trauma, and degenerative cases revealed an important decrease from 2019 to 2020. Among complex procedures, a decrease was noted in corpectomy associated to a complete discontinuation of deformity surgeries.

In the entire series comprising 62 patients, only one tested COVID+ preoperatively and underwent surgery with the previously described protections measures [5].

Whenever possible (47\%), minimal-invasive procedures
Table 3. Number of simple spine procedures during the same period of time (March 17 to April 17) in 2019 and 2020

\begin{tabular}{lrr} 
& \multicolumn{2}{c}{ Year } \\
\cline { 2 - 3 } Simple surgical procedures & 2019 & 2020 \\
\hline Thoracic disc herniation & 0 & 1 \\
\hline Lumbar disc herniation & 12 & 15 \\
\hline Cervical disc herniation & 21 & 4 \\
\hline Lumbar canal narrowing & 22 & 7 \\
\hline Foraminotomy & 0 & 4 \\
Wound infections & 10 & 3 \\
(with or without hardware removal) & & \\
\hline Biopsy & 0 & 2 \\
\hline Total & 65 & 36 \\
\hline
\end{tabular}

Table 4. Number of intermediate spine procedures during the same period of time (March 17 to April 17) in 2019 and 2020

\begin{tabular}{lcc} 
& \multicolumn{2}{c}{ Year } \\
\cline { 2 - 3 } Intermediate procedures & 2019 & 2020 \\
Short segment fixation & 29 & 14 \\
\hline Balloon kyphoplasty (tumor cases) & 5 & 4 \\
$\begin{array}{l}\text { Vertebral cement expansion procedures } \\
\text { (trauma cases) }\end{array}$ & 22 & 5 \\
Transforaminal lumbar interbody fusion & 10 & 1 \\
\hline Total & 66 & 24 \\
\hline
\end{tabular}

Table 5. Number of complex spine procedures during the same period of time (March 17 to April 17) in 2019 and 2020

\begin{tabular}{lcc} 
& \multicolumn{2}{c}{ Year } \\
\cline { 2 - 3 } Complex procedure & 2019 & 2020 \\
Post-traumatic deformity & 1 & 3 \\
Vertebrectomy & 6 & 3 \\
Deformity & 8 & 0 \\
Total & 15 & 6 \\
\hline
\end{tabular}

were preferentially chosen with percutaneous fixation and decompression using tubular retractors. In order to reduce the surgical time, surgeries were performed mostly by senior surgeons ( $80 \%$ in 2020 versus $60 \%$ in 2019) and with two senior operators for complex cases.

During the follow-up period, two patients were tested COVID+ during the postoperative course, one required 
to be transferred to a dedicated ward for isolation and treatment for 8 days, while the other only required classic medical surveillance using dedicated protective measures. No cases of contamination in medical or paramedical staff were reported using PCR-testing.

\section{Discussion}

The actual sanitary crisis led to a massive mobilization of the sanitary system toward intensive care units and management of COVID-19 patients. This unstable period is therefore drastically reducing the possibility for surgical procedures. However, some patients still require spinal interventions. In such cases, many adaptations in the health care structures are necessary with specific isolated rooms and operative theaters for COVID+ patients and the need for perfect coordination between all healthcare professionals $[6,7]$.

According to Zou et al. [5], the first step of management is related to screening for COVID-19 symptoms and triage of the patients that would require a surgical procedure. In our experience, we used systematic preoperative PCR-testing and reserved chest-CT-scan for suspected cases. However, as stated recently by Sornsa-Ard et al. [8], preoperative testing is not always possible with regard to the clinical situation of the patient, especially for trauma cases. In such cases, full protective measures are necessary until results are available.

Results from our experience revealed that despite a drastic decrease in the number of surgical spine procedures, it is still necessary to provide care to patients for specific conditions. In addition to scientific guidelines, it is important to remember that each situation is unique, and many parameters need to be considered. Some factors are related to the patient; however, most are associated to the pandemic conditions. Spine surgeons are an integral part of the global organization who are responsible for the selection of appropriate surgical indications. Results from this study revealed that tumor and infectious cases were stable between 2019 and 2020, while trauma cases drastically reduced, probably due to confinement of the population with a concomitant reduction of sports and motor vehicles accidents. With regard to degenerative cases, a global decrease was observed that appears logical as it represents chronic conditions. However, we do not have clear explanations for the drastic decrease in the cases of cervical disc herniation. When considering complex surgeries that may require postoperative ICU resources, indications must be limited to urgent cases only. Further, as in our experience, deformity cases are rescheduled to after the pandemic.

This study has certain limitations related to its design and the relatively short observation period. However, it underlines the need for perfect cooperation among healthcare specialists. Our institution was located in a medium-low cluster region of COVID that did not lead to saturation of the healthcare system. Therefore, it was possible to organize a specific organization; however, this may not be possible in high COVID cluster zones.

\section{Conclusions}

During the COVID-19 pandemic, it is possible to maintain spine surgical activity as per various parameters. Each surgical procedure must be discussed and organized with all the caregivers who are involved, such as surgeons, anesthetists, and infectious diseases physicians. Indications for surgery must be in line with the scientific guidelines and should be adapted to each healthcare facility. Whenever possible, minimal-invasive strategies must be preferred to limit the need for postoperative intensive care unit resources. Complex surgeries should ideally be performed by two senior surgeons.

\section{Conflict of Interest}

No potential conflict of interest relevant to this article was reported.

\section{Author Contribution}

S. Prost, K. Farah, M. Meyer, and JB. Denis wrote the manuscript. S. Fuentes, H. Dufour, and B. Blondel revised the manuscript and coordinate research.

\section{References}

1. World Health Organization. WHO Director-General's opening remarks at the media briefing on COVID-19. Geneva: World Health Organization; 2020.

2. Ministere des Solidarites et de la Sante. Preparing for the COVID-19 epidemic risk: methodological guide. Paris: Ministere des Solidarites et de la Sante; 2020.

3. Agence Regionale de Sante Provence Alpes Cote 
d'Azur. Coronavirus: update on March 16, 2020. Marseille: Agence Regionale de Sante Provence Alpes Cote d'Azur; 2020.

4. Societe Francaise de Chirugie du Rachis (SFCR). Modalities of treatment of the spinal surgery patients in the period of COVID-19 related crisis. Paris: Societe Francaise de Chirugie du Rachis (SFCR); 2020.

5. Zou J, Yu H, Song D, Niu J, Yang H. Advice on standardized diagnosis and treatment for spinal diseases during the coronavirus disease 2019 pandemic. Asian Spine J 2020;14:258-63.
6. Wong J, Goh QY, Tan Z, et al. Preparing for a COVID-19 pandemic: a review of operating room outbreak response measures in a large tertiary hospital in Singapore. Can J Anaesth 2020 Mar 11 [Epub]. https://doi.org/10.1007/s12630-020-01620-9.

7. Coccolini F, Perrone G, Chiarugi M, et al. Surgery in COVID-19 patients: operational directives. World J Emerg Surg 2020;15:25.

8. Sornsa-Ard T, Niramitsantiphong A, Liawrungrueang $\mathrm{W}$. Management of traumatic spinal fracture in the coronavirus disease 2019 situation. Asian Spine J 2020 Apr 24 [Epub]. https://doi.org/10.31616/ asj.2019.0183. 